\title{
INVITED PAPER \\ ISH2022: New Normal ISH Meeting Born in Kyoto
}

\author{
HIROSHI ITOH \\ President of ISH2O22 \\ Keio University School of Medicine Tokyo, Japan \\ President, The Japanese Society of Hypertension, Vice president of ISH
}

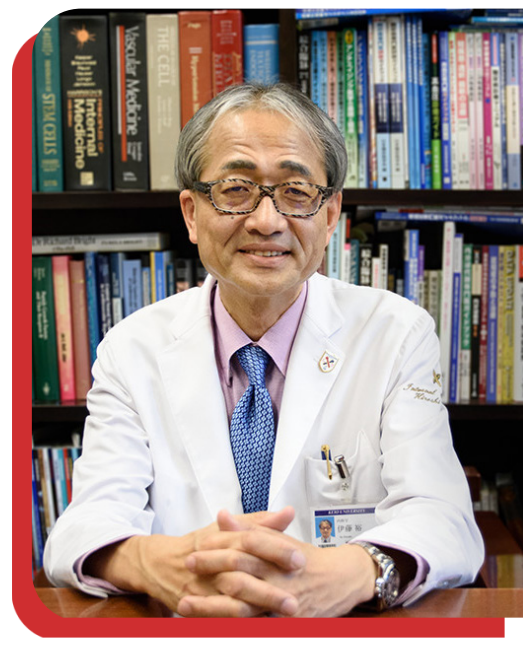

\section{DOI:10.30824/2106-16}

ISH2022 will be held from October 12 to 16 2022 in the City of Kyoto at Kyoto International Conference Center with the theme of "The Wisdom for Conquering Hypertension". Please save the date!

Although COVID-19 forced us to meet virtually for a while, we hope ISH2022 Kyoto will be a chance for many of our colleagues and friends from all over the world to gather in together in person and share our wisdom on conquering hypertension to ensure a better quality of life for people.

We will also provide ISH2022 programs in a virtual format for those who have limited access, to offer expanded opportunities.

Why should you attend ISH2O22 in Kyoto?

It's because we:

\section{Provide cutting-edge innovative scientific programs \\ 2. Offer the opportunity to explore the old capital of Japan and knowledge-centered city, Kyoto \\ 3. Provide a variety of interesting, informative and interactive sessions for many young and enthusiastic clinicians and researchers}

\section{Innovative scientific programs}

We are honored to have the 2012 Nobel Prize Laureate for the discovery of iPS cells, Shinya Yamanaka for the keynote lecture at ISH2022! Not only that, ISH2022 covers the following categories;

\section{Three Main Topics: Al/Dx, FOOD/ Nutrition, Physical Fitness with the view of correlations with hypertension}

We believe that these three topics have interdisciplinary impacts correlations and will lead to the realization of well-being of all the people in the world.

\section{Hypertension for SDGs Toward 2030 World (Climate, Disaster, Isolation/Mental health etc.)}

Hypertension and hypertension-related diseases are relevant to many of sustainable development goals including the problems of poverty and hunger, education, gender, disaster, and climate change. We discuss and share the information about hypertension and hypertension-related diseases from the perspectives of SDGs

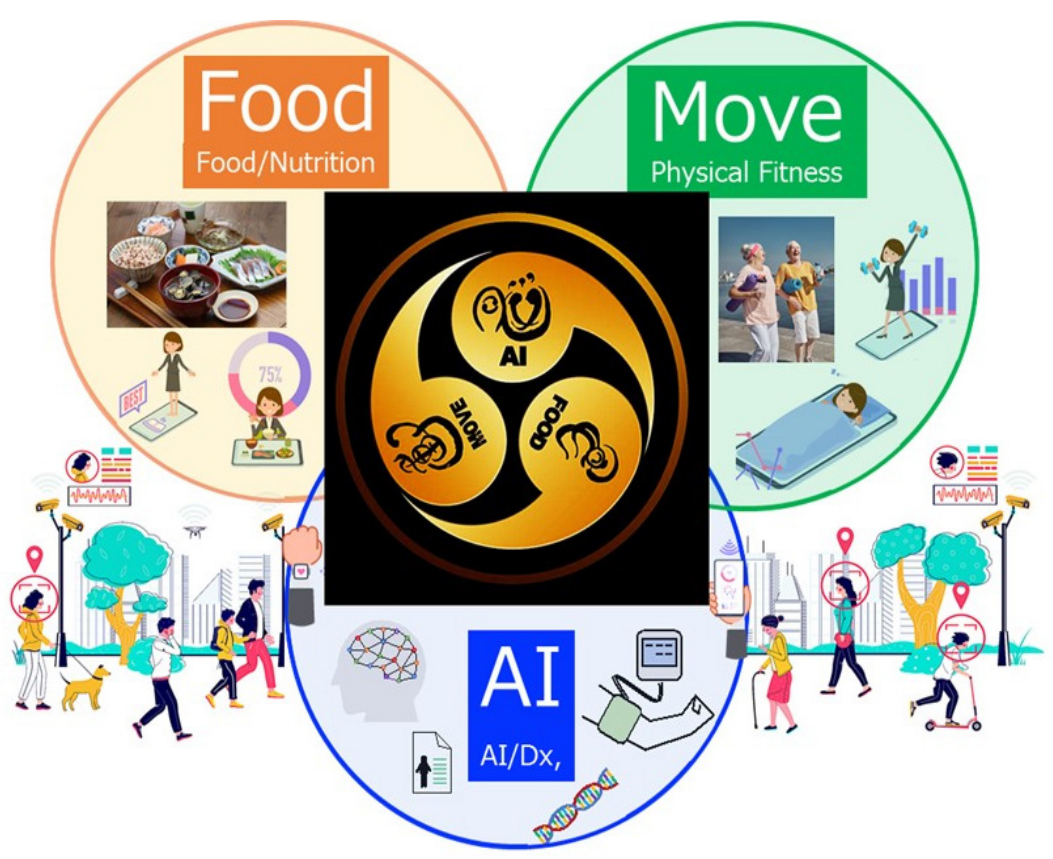

3. Global Health and Hypertension with Diversity (Racial, Economical difference, Medical resources etc.)

Considering racial and social differences of the environment in terms of education and lifestyle, pathogenesis and epidemiology of hypertension are diverse. Therefore, prevention and treatment of hypertension to achieve a better quality of life need to be approached with their diversities. 


\section{Life-course and Hypertension (Preconception, DOHaD, cancer etc. and hypertension)}

The significance of hypertension differs at each stage of life. At each stage, a multifaceted approach is required. Hypertension control and research need a seamless transition from one stage to another.

\section{Super-aged Society and Hypertension (Sarcopenia, Dementia, Social Capitals etc.)}

Japan is now a super-aged society and coping with the increase of aging population is an urgent issue. The number 1 cause of death from non-communicable diseases is hypertension. In super-aged societies, prevention and control of hypertension is essential for healthy and happy longevity.

\section{Hypertension Next Generation therapy (Renal Denervation, Single compound pills, Applications etc.)}

Since many target molecules for antihypertensive therapy are being identified, new antihypertensive drugs are expected to be developed. Furthermore, challenging antihypertensive therapies such as device therapy, vaccines or apps. are being developed. We will discuss the current status of the development of next-generation hypertension treatment methods and their future directions.

\section{Convergence of Communicable Diseases and NCDs}

Hypertension has been categorized as a lifestyle-related disease, i.e., non-communicable diseases (NCDs), but the COVID-19 pandemic has opened our eyes for the need to restructure our understanding of it and its relations with communicable diseases.

\section{Japan Method for conquering hypertension ("Hypertension Zero Town" etc.)}

We will introduce JSH achievements aiming at the creation of "Hypertension Zero Town" in cooperation with more than ten Japanese cities and towns nationwide with the aid of Japanese Government by various innovative methods.

\section{Pathophysiology of Hypertension: Chronicle to the Future}

We will review the past and future prospects of hypertension research, which aims at the deep understanding of pathophysiology.

\section{Blood Pressure Measurement: Conventional and Future}

The history of clinical research and medical practice of hypertension began nearly 100 years ago, with the first development of blood pressure measurement. Now, wearable devices enable us to monitor blood pressure for 24 hours, real time. We will discuss how such methods have changed and will change the future of hypertension, its direction, possibilities and challenges.

\section{Imaging and Biomarker for Hypertension Management}

Imaging and biomarkers play an important role in the evaluation of the degree and evolution of various organ damages caused by hypertension. The establishment of more accurate testing methods with non-invasive approaches is essential for the realization of preemptive medicine and personalized medicine for hypertension.

\section{Hypertension Reigning Over Systemic Diseases}

By understanding the dynamic link between hypertension and systemic diseases such as stroke, cardiovascular diseases, heart failure, diabetes, and metabolic syndrome etc., we can expect to develop new medicine for hypertension.

\section{Kyoto: Old capital of Japan and Knowledge- centered city}

Kyoto, the ancient capital of Japan, is famous for its long history and unique culture.

Kyoto has 17 UNESCO World Cultural Heritage Sites and is brimming with unique culture and atmosphere. Kyoto is also renowned as one of the most academic cities in Japan. Including Kyoto University, one of the most prestigious universities in Japan, Kyoto has 50 universities and colleges that provide higher and specialized education to Japanese as well as overseas students. Kyoto University is the birthplace of Spontaneously Hypertensive Rats (SHR), which were first 
